\title{
Fell off of a horse - journey from Emergency Department to Stroke clinic
}

\author{
E J Traer, T Loganathan, D M Sinha, P C Guyler, A O’Brien \\ Department of Stroke Medicine, Southend University Hospital, Southend, UK
}

Correspondence to P C Guyler, emmatraer@doctors.org.uk

\section{Summary}

The authors present a case of a young woman who presented with transient episodes of left-sided weakness after she fell off a horse. She attended Emergency Department twice before being referred to the Stroke clinic, where she was diagnosed with carotid artery dissection.

\section{BACKGROUND}

Emergency physicians should consider the possibility of arterial dissection in any trauma patient with headache or neurological signs, when consideration of common causes fails to lead to a diagnosis. Awareness of its clinical features and advances in imaging in recent times can lead to earlier detection of this condition. Failure to suspect a dissection in minor injury can lead to permanent neurological deficits.

Traumatic injury to the major vessels of the head and neck can result in potentially devastating neurological sequelae. ${ }^{1}$ Traumatic carotid dissection occurs in approximately $1 \%$ of all patients with blunt injury mechanisms and is frequently initially unrecognised. ${ }^{2}$ Cervical dissection accounts for $20-30 \%$ of all ischaemic strokes in patients younger than 50 years of age. Carotid and vertebral arterial dissection can present with pain, headache, Horner's syndrome, stroke or transient ischaemic attack (TIA).
Risk factors for dissection include trauma, connective tissue disorders, hypertension, smoking, diabetes mellitus, hyperlipidaemia and the combined contraceptive pill.

A case of a young woman who presented with transient episodes of left-sided weakness and diagnosed with carotid artery dissection is reported here.

\section{CASE PRESENTATION}

A fit 54-year-old woman with an unremarkable past medical history was seen in Accident and Emergency (A\&E) on 2 consecutive days with left-sided facial and arm numbness and left-arm weakness. On both occasions, she was discharged home from A\&E, but was referred to the TIA clinic after her second $A \& E$ attendance. She was seen 1 week later in the TIA clinic, and, on further questioning, she reported that the first episode completely resolved in 20-30 min and the second after $30-60 \mathrm{~min}$. She also gave a history of a fall

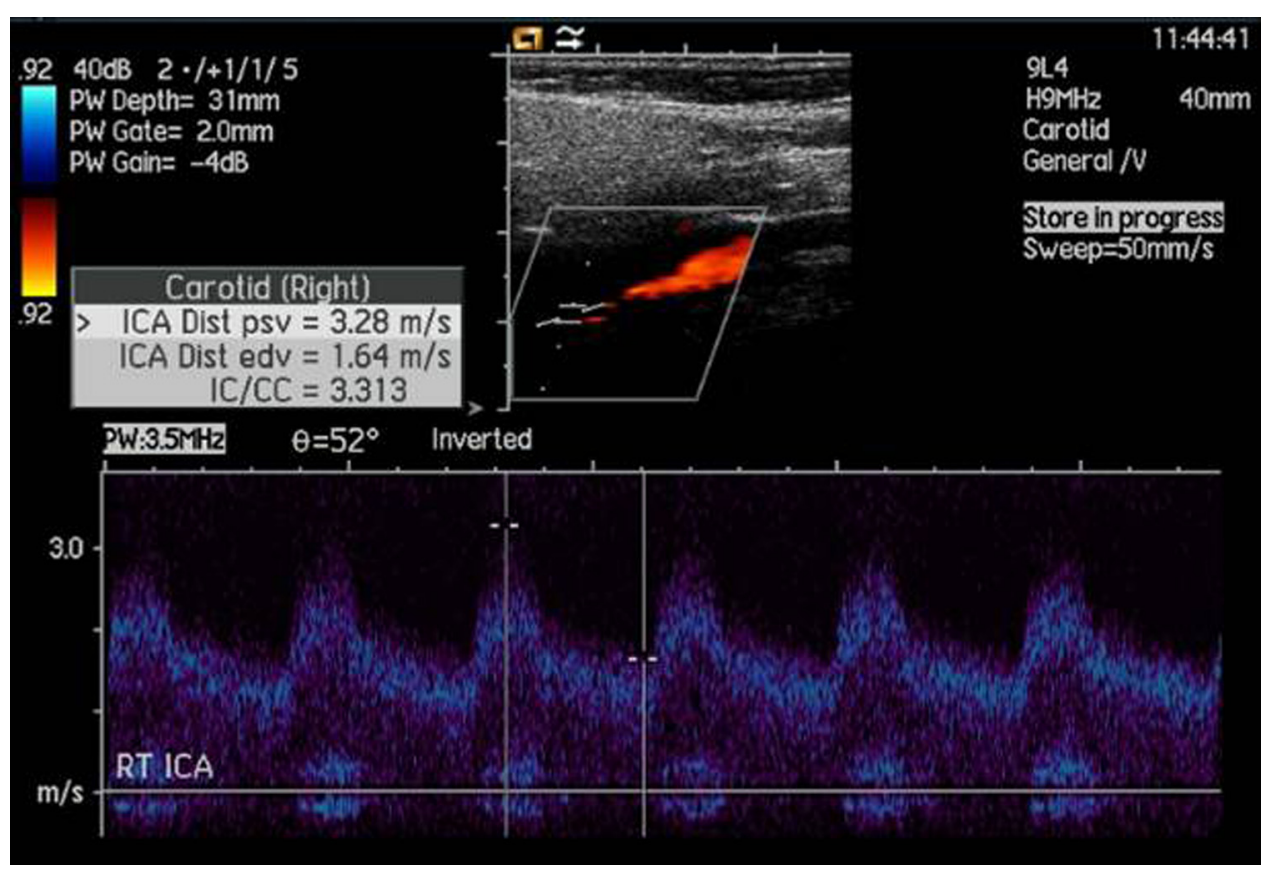

Image 1 Carotid Doppler's showing 70\% stenosis of the right internal carotid artery. 


\section{BMJ Case Reports}

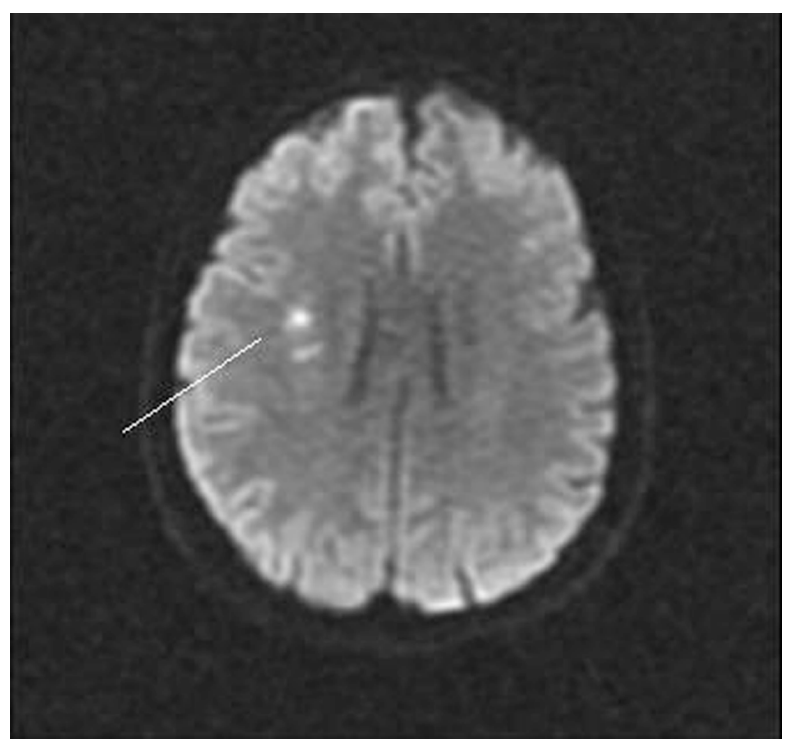

Image 2 MRI DWI scan showing two areas of recent infarction seen in the right corona radiata.

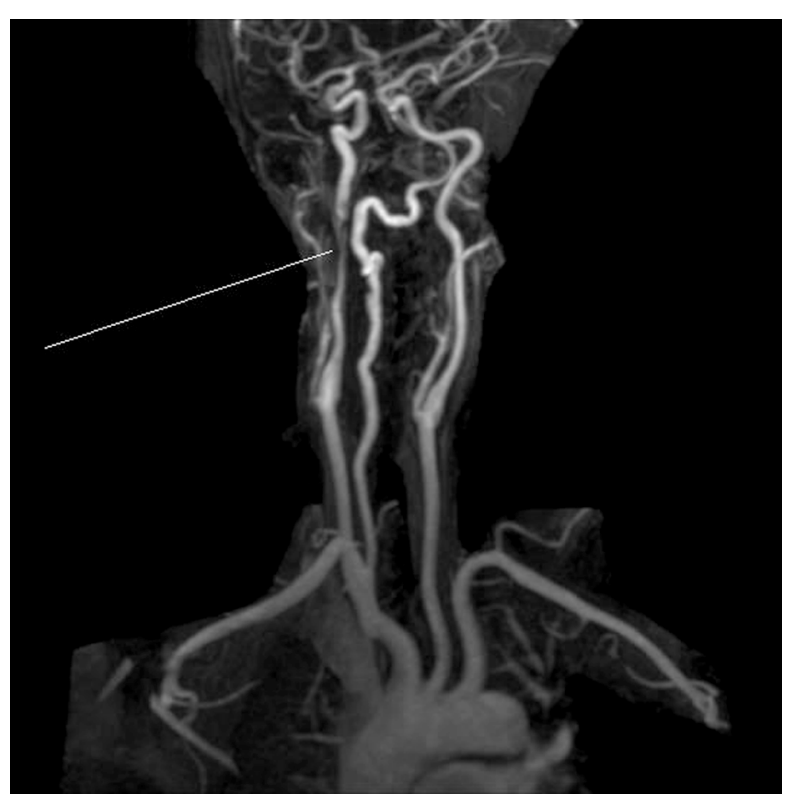

Image 4 MRA scan showing right internal carotid artery dissection and $80 \%$ narrowing.

from her horse 2 weeks previous resulting in right-hip pain. She denied any head injury or neck pain. Cardiorespiratory and neurological examinations were unremarkable.

\section{INVESTIGATIONS}

Her carotid Doppler (image 1) showed $70 \%$ stenosis of the right internal carotid artery. Her MRI (images 2 and 3) and magnetic resonance arteriogram (images 4 and 5) showed two areas of recent infarction seen in the right corona radiata/centrum semi-ovale and right internal carotid artery dissection and severe (80\%) narrowing.

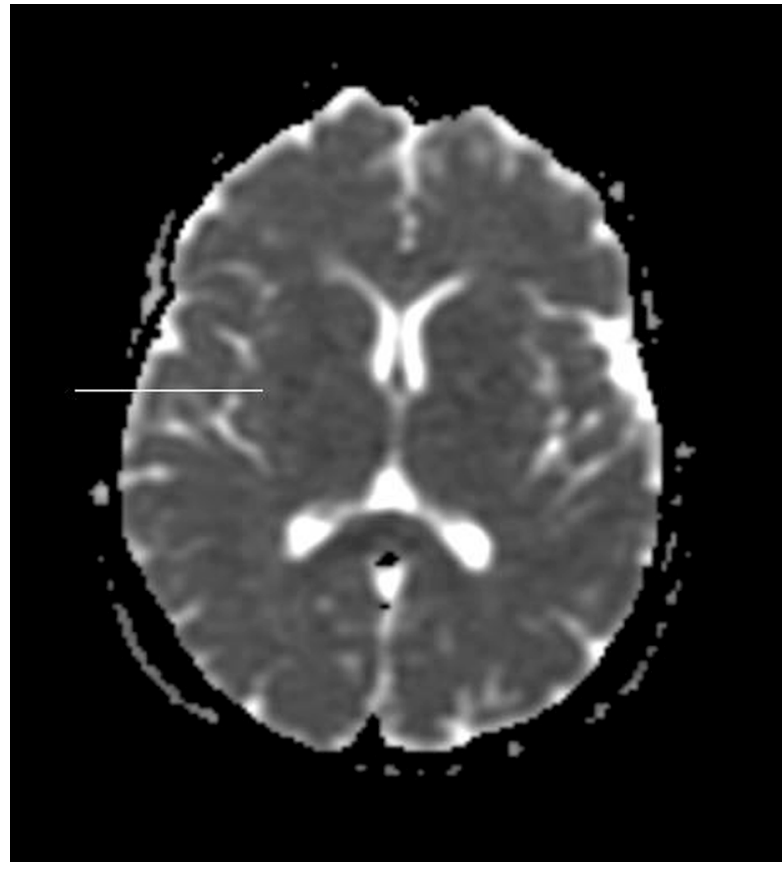

Image 3 MRI ADC scan showing corresponding two areas of recent infarction.

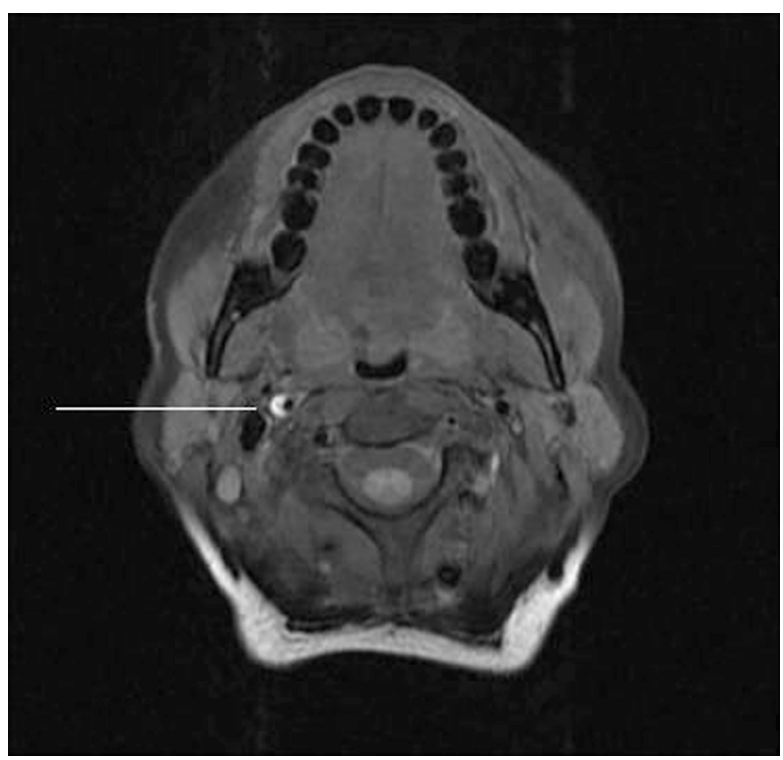

Image 5 MRA scan showing crescent of right internal carotid artery dissection.

\section{TREATMENT}

The patient was treated with antiplatelet drugs.

NICE guidelines recommend that people with stroke secondary to acute arterial dissection should be treated with either anticoagulants or antiplatelet agents, preferably as part of a randomised controlled clinical trial to compare the effects of the two treatments. ${ }^{3}$ The CADISS Study (Cervical Artery Dissection In Stroke Study) is an ongoing, randomised, multi-centre, prospective study to determine 


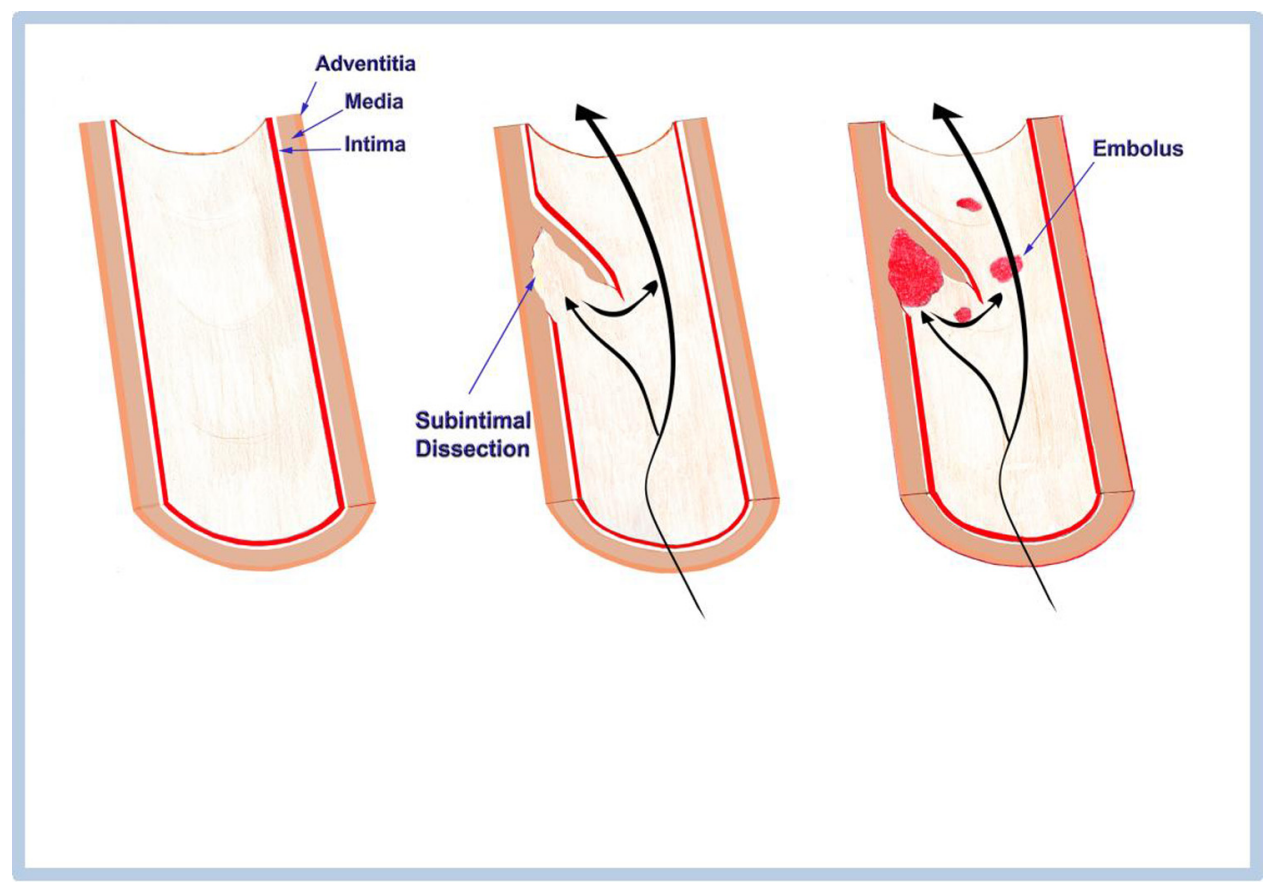

Figure 1 Illustration of arterial dissection.

whether antiplatelet agents or anticoagulation are more effective at preventing stroke in patients with acute cervical dissection. ${ }^{4}$ However, the patient presented to the Stroke team too late to meet the inclusion criteria to enter this trial.

\section{OUTCOME AND FOLLOW-UP}

The patient is to be followed up with a repeat MRI scan after 3 months.

Carotid artery dissection generally carries an excellent prognosis if treatment is initiated early. ${ }^{5}$ Many clinician's anticoagulate patients present with cervical dissection for 3-6 months. However, this is not evidence based, and there is currently no evidence base to guide treatment. The CADISS study is an ongoing, randomised, multi-centre, prospective study to determine whether antiplatelet agents or anticoagulation are more effective at preventing stroke in patients with acute cervical dissection. ${ }^{4}$

\section{DISCUSSION}

Carotid artery dissection accounts for $20-30 \%$ of all ischaemic strokes in patients younger than 50 years of age ${ }^{4}$ and is the underlying mechanism in $2.5 \%$ of all strokes. ${ }^{6}$ There is a high risk of recurrent stroke within the first 4 weeks; however, after this recurrence is rare (about 1\% per year).

Carotid artery dissection can occur intracranially and extracranially, with the latter being more frequent. Internal carotid artery dissection can be a result of mechanical forces such as trauma or stretching ${ }^{7}$ as well as underlying connective tissue disorders such as Ehlers-Danlos syndrome IV. $^{4}{ }^{9}$ Other risk factors include hypertension, hyperlipidaemia, diabetes mellitus, smoking and the combined contraceptive pill. ${ }^{4}$
Arterial dissection begins as a tear in the tunica intima or between the tunica media and tunica adventitia and then under arterial pressure; blood dissects along the artery, resulting in an intramural haematoma (figure 1). This process can result in stenosis or thromboemboli and lead to ischaemic stroke; also, aneurismal outpouchings may compress the lower cranial nerves. 59

A high degree of suspicion should be taken in to account even after low-impact trauma. Ten investigations are considered mandatory in the presence of (1) a potential arterial origin haemorrhage from the nose, ears, mouth or a wound; (2) an expanding cervical haematoma; (3) cervical bruit in a patient $>50$ years of age; (4) evidence of acute infarct at brain imaging; (5) unexplained central or lateralising neurological deficit or TIA; or (6) Horner's syndrome, neck or head pain. A number of centres screen asymptomatic patients with blunt trauma for dissection.

Colour duplex ultrasound, CT, CT angiogram and magnetic resonance angiography are non-invasive screening methods. Catheter angiography is the gold standard but is rarely required for diagnosis. ${ }^{10}$

Prompt hospital referral is essential while assessing disability in young-age patients, and thrombolysis with rT-PA (Alteplase) should be considered in this group of patients, regardless of dissection, as would be the case in treating any other ischemic stroke. Despite a common fear of thrombolysis in this group, recent studies indicate that thrombolysis should not be excluded in patients who might have carotid artery dissection. ${ }^{11}$

Rarely, endovascular therapy may be indicated for the treatment of ruptured aneurysms or to prevent recurrent ischaemia. ${ }^{5}$ Endovascular treatment of traumatic internal carotid artery injury continues to evolve. ${ }^{12}{ }^{13}$ 


\section{BMJ Case Reports}

Thromboarterectomy, direct suture of intimal tears and extracranial-intracranial bypass should be considered in exceptional cases. ${ }^{10}$

Patients with dissection-related cervical artery occlusion have a significantly increased risk of suffering a disabling stroke. ${ }^{14}$ This case highlights the importance of recognising the possible causes of TIA/stroke in the young- and middleage groups in Emergency departments.

\section{Learning points}

-When a young- or middle-aged person, without vascular risk factors, presents with symptoms of TIA/ stroke, dissection must be ruled out.

- There are various possible presentations of carotid artery dissection.

- High-risk TIAs should be admitted, where available, to acute stroke units.

- Prompt hospital referral is essential for looking at disability in young age and thrombolysis should be considered.

\section{Competing interests None}

Patient consent Obtained.

\section{REFERENCES}

1. Redekop GJ. Extracranial carotid and vertebral artery dissection: a review. Can J Neurol Sci 2008;35:146-52.
2. Nedeltchev K, Baumgartner RW. Traumatic cervical artery dissection. Front Neurol Neurosci 2005;20:54-63.

3. NICE Guidelines. 83 Antiplatelet and Anticoagulation Treatment Due to Arterial Dissection, 10 January 2008. http://www.nice.org.uk/guidance/ index.jsp?action $=$ download\&o $=38915$.

4. Markus H, Norris J. CADISS: Cervical Artery Dissection in Stroke Study. St George's: University of London.

5. DuBose J, Recinos G, Teixeira PG, et al. Endovascular stenting for the treatment of traumatic internal carotid injuries: expanding experience. Trauma 2008;65:1561-6.

6. Lyrer $\mathbf{P}$, Engelter S. Antithrombotic Drugs for Carotid Artery Dissection. The Cochrane Collaboration Cochrane Reviews, 1 April 2003. http:// www.cochrane.org/reviews/en/ab000255.html.

7. Haneline MT, Rosner AL. The etiology of cervical artery dissection. J Chiropr Med 2007:6:110-20.

8. Baker WE, Servais EL, Burke PA, et al. Blunt carotid injury. Curr Treat Options Cardiovasc Med 2006:8:167-73.

9. Zohrabian D, Leber MJ. Dissection, Carotid Artery. 21 October 2008. http:// emedicine.medscape.com/article/757906-overview.

10. Abou-Chebl A. Cervical artery dissection. Curr Treat Options Cardiovasc Med 2009;11:167-75

11. Engelter ST, Rutgers MP, Hatz F, et al. Intravenous thrombolysis in stroke attributable to cervical artery dissection. Stroke 2009;40:3772-6.

12. Dziewas R, Konrad C, Drôger B, et al. Cervical artery dissection-clinical features, risk factors, therapy and outcome in 126 patients. J Neurol 2003;250:1179-84.

13. Fava $\mathbf{M}$, Meneses $L$, Loyola $\mathrm{S}$, et al. Carotid artery dissection: endovascular treatment. Report of 12 patients. Catheter Cardiovasc Interv 2008;71:694-700.

14. Baker WE, Wassermann J. Unsuspected vascular trauma: blunt arterial injuries. Emerg Med Clin North Am 2004;22:1081-98.

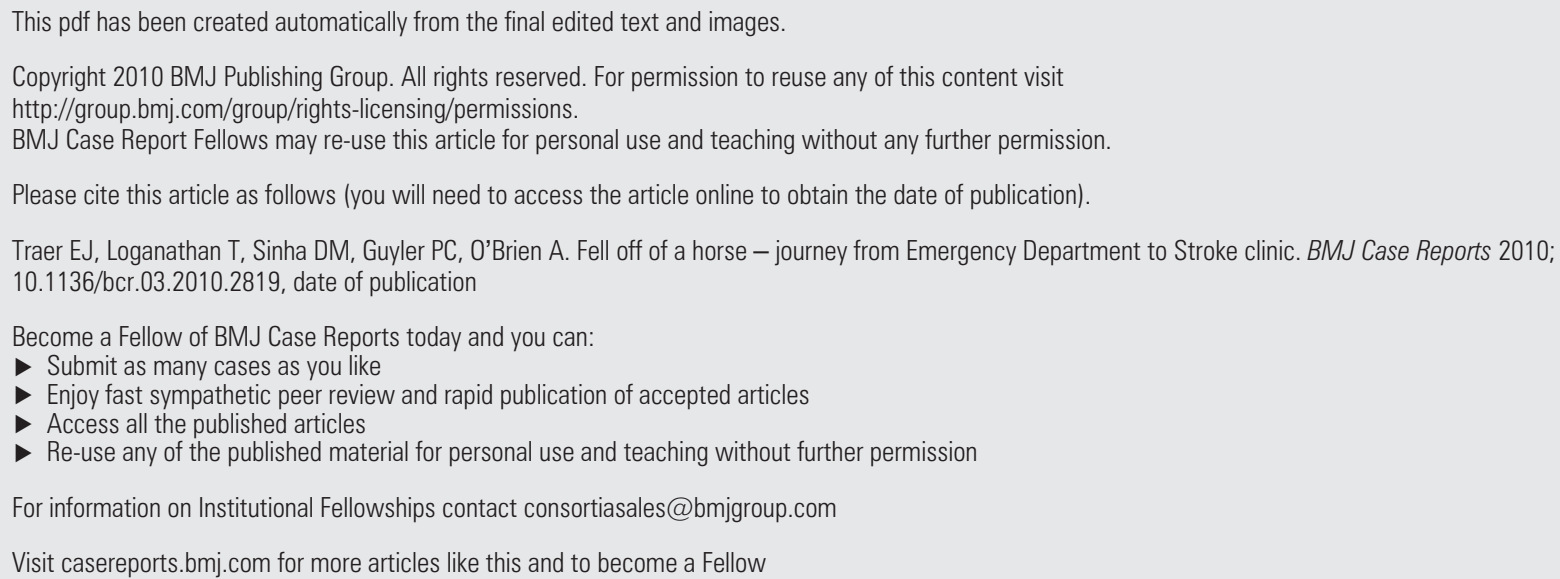

